\title{
MONOTONICITY OF THE PRINCIPAL EIGENVALUE OF THE $p$-LAPLACIAN IN AN ANNULUS
}

\author{
B. EMAMIZADEH AND M. ZIVARI-REZAPOUR \\ (Communicated by Walter Craig)
}

\begin{abstract}
In this note we prove a monotonicity result related to the principal eigenvalue of the $p$-Laplacian in an annulus in $\mathbb{R}^{N}$.
\end{abstract}

\section{INTRODUCTION}

This note is motivated by [3], where the authors consider the principal eigenvalue for $-\Delta$, with respect to the Dirichlet boundary conditions, in $D(h):=B \backslash B_{h}$. Here $B$ stands for the unit ball in $\mathbb{R}^{N}$ centered at the origin, and $B_{h}$ is the ball centered at $(h, 0) \in \mathbb{R} \times \mathbb{R}^{N-1}$ with radius $a<1$. They show the principal eigenvalue as a function of $h$ decreasing over the interval $[0,1-a]$.

In this work we show a similar result, but for $-\Delta_{p}$. For $p \in(1, \infty), \Delta_{p}$ denotes the usual p-Laplacian operator; that is $\Delta_{p} u=\nabla \cdot\left(|\nabla u|^{p-2} \nabla u\right)$. Let us describe the problem. We fix $-1<a_{0}<0$, and consider $f \in L^{\infty}(B)$. We assume $f^{+}(x):=\max (f(x), 0)$ is supported in $S:=\left\{x \in B: x_{1}<a_{0}\right\}$ and does not vanish identically. Also, we assume $f^{-}:=f^{+}-f$ is non-increasing in the $x_{1}$-variable. Let us consider the following eigenvalue problem:

$$
\text { (BVP) } \begin{cases}-\Delta_{p} u=\lambda f(x)|u|^{p-2} u & \text { in } D(h), \\ u=0 & \text { on } \partial D(h) .\end{cases}
$$

By $\lambda(h)$ we denote the principal eigenvalue of (BVP), and by $u(h)$ the corresponding positive (principal) eigenfunction normalized by

$$
\int_{D(h)} f(x) u^{p}(h) d x=1 .
$$

It is well known that $\lambda(h)$ is simple, $u(h) \in C^{1}(\overline{D(h)})$, and $\partial u(h) / \partial \nu<0$ on $\partial D(h)$, where $\nu$ stands for the unit outward normal on $\partial D(h)$; see for example [2]. The main result of the paper is the following

Theorem 1. The principal eigenvalue $\lambda(h)$ is decreasing on $\left[-a_{0}, 1-a\right]$.

The idea of the proof of Theorem 1 is straightforward; we show the derivative of $\lambda(h)$ is non-positive on $\left[-a_{0}, 1-a\right]$. This is the same idea employed in [3], however

\footnotetext{
Received by the editors December 13, 2006 and, in revised form, February 19, 2007 and February $28,2007$.

2000 Mathematics Subject Classification. Primary 35P30.

Key words and phrases. $p$-Laplacian, principal eigenvalue, domain derivative.
} 
in our case the technicalities are very different. In particular we use the concept of domain derivative to a great extent, and the reference we use for this is [4].

\section{Domain DeRIVATIVE}

Throughout this section $\Omega$ denotes a bounded smooth domain in $\mathbb{R}^{N}$. For a vector field $V \in C^{2}\left(\mathbb{R}^{N}, \mathbb{R}^{N}\right)$, and non-negative $t$, we denote by $\Omega_{t}$ the image of $\Omega$ under the map $I+t V$. It is well known that when $t$ is small the map $I+t V$ is a diffeomorphism, hence $\Omega_{t}$ would be as "good" as $\Omega$, roughly speaking.

Suppose $\mathcal{L}$ is a differential operator that maps $W^{m, p}\left(\Omega^{\prime}\right)$ into $\mathcal{D}^{\prime}\left(\Omega^{\prime}\right)$, the space of distributions on $\Omega^{\prime}$, where $\Omega^{\prime}$ is any open set inside $\Omega$. Suppose $w$ is the solution of the following boundary value problem:

$$
\begin{cases}\mathcal{L} u=0 & \text { in } \Omega^{\prime} \\ u=0 & \text { on } \partial \Omega^{\prime}\end{cases}
$$

Also suppose $w^{t}$ is the solution of (2) with $\Omega^{\prime}$ replaced by $\Omega_{t}^{\prime}$. Then the function $w^{\prime}$ defined on $\Omega^{\prime}$ by

$$
w^{\prime}(x)=\lim _{t \rightarrow 0^{+}} \frac{w^{t}\left(x^{t}\right)-w(x)}{t}
$$

where $x^{t}:=x+t V(x)$ is called the domain derivative of $w$ at $\Omega^{\prime}$ in the direction of $V$. In practice $w^{\prime}$ usually satisfies

$$
\begin{cases}\frac{\partial \mathcal{L}}{\partial u} w^{\prime}=0 & \text { in } \Omega^{\prime} \\ w^{\prime}=-\frac{\partial w}{\partial \nu} V \cdot \nu & \text { on } \partial \Omega^{\prime}\end{cases}
$$

where $\nu$ stands for the unit outward normal to $\partial \Omega^{\prime}$. The reader is referred to [4] for a thorough discussion on domain derivatives. As an example we consider the (BVP) introduced in the previous section. For simplicity we write $u$ and $\lambda$ in place of $u(h)$ and $\lambda(h)$, respectively. Then we have the following result

Lemma 1. With the notation introduced above, $u$ satisfies

$$
\left\{\begin{array}{rlrl}
-\nabla & \cdot\left((p-2)|\nabla u|^{p-1} \frac{\nabla u \cdot \nabla u^{\prime}}{|\nabla u|^{3}} \nabla u+|\nabla u|^{p-2} \nabla u^{\prime}\right) & & \\
& =\lambda^{\prime} f(x) u^{p-1}+(p-1) \lambda f(x) u^{p-2} u^{\prime} & & \text { in } D(h), \\
u^{\prime}=-\frac{\partial u}{\partial \nu} V \cdot \nu & & \text { on } \partial D(h) .
\end{array}\right.
$$

Proof. The boundary equation in (3) follows directly from [4, Theorem 3.2, p. 664], and the right hand side of the differential equation follows from the product rule for domain derivatives, noting that $f(x)$ does not depend on the domain. To derive the left hand side of the differential equation we apply [4, Theorem 3.1, p. 663]. Indeed since the domain differential operator commutes with the divergence operator we only need to show

$$
\left(|\nabla u|^{p-2} \nabla u\right)^{\prime}=(p-2)|\nabla u|^{p-1} \frac{\nabla u \cdot \nabla u^{\prime}}{|\nabla u|^{3}} \nabla u+|\nabla u|^{p-2} \nabla u^{\prime},
$$

where the "prime" denotes the domain derivative. The identity

$$
|\nabla u|^{p-2} \nabla u=(\nabla u \cdot \nabla u)^{\frac{p-2}{2}} \nabla u
$$


is clear, so when we differentiate both sides, applying the product rule to the right hand side, we obtain

$$
\begin{aligned}
\left(|\nabla u|^{p-2} \nabla u\right)^{\prime} & =\frac{p-2}{2}(\nabla u \cdot \nabla u)^{\frac{p-2}{2}-1}\left(\nabla u^{\prime} \cdot \nabla u+\nabla u \cdot \nabla u^{\prime}\right) \nabla u \\
& +(\nabla u \cdot \nabla u)^{\frac{p-2}{2}} \nabla u^{\prime} \\
& =(p-2)|\nabla u|^{p-1} \frac{\nabla u \cdot \nabla u^{\prime}}{|\nabla u|^{3}} \nabla u+|\nabla u|^{p-2} \nabla u^{\prime},
\end{aligned}
$$

as desired.

\section{Proof of Theorem 1}

In this section we prove Theorem 1, for which we need the following "weak comparison principle".

Theorem 2. Let $\Omega \subset \mathbb{R}^{N}$ be a bounded domain with piecewise smooth boundary $\partial \Omega$. Suppose $\omega$ is an open smooth subset of $\partial \Omega$. Suppose $g: \Omega \times[0, \infty) \rightarrow[0, \infty)$ is a Carathèodory function such that $g(x, \cdot)$ is non-decreasing for every $x \in \Omega$. Let $v, u \in C^{1}(\bar{\Omega})$ be non-negative weak solutions of the following differential inequalities:

$$
\left\{\begin{array}{l}
-\Delta_{p} v+g(x, v) \leq 0 \\
-\Delta_{p} u+g(x, u) \geq 0
\end{array}\right.
$$

in $\Omega$, respectively. Suppose $u=v$ on $\omega$ and $v \leq u$ on $\partial \Omega \backslash \omega$. Also, suppose $\partial u / \partial \nu<0$ on $\omega$. Then there is a neighborhood of $\omega$, denoted $\mathcal{N}(\omega) \subset \Omega$, such that $u \geq v$ in $\mathcal{N}(\omega)$.

Proof. It is clear that if $\{x \in \Omega: v(x)>u(x)\}$ is empty, then the assertion of the theorem follows trivially; so we assume otherwise. It suffices to show that the boundary of the open set $\{x \in \Omega: v(x)>u(x)\}$ does not intersect $\omega$. Note that since $u \geq v$ on $\partial \Omega,(v-u)^{+} \in W_{0}^{1, p}(\Omega)$; thus it can be used as a test function in both inequalities in (4). After multiplying each of the inequalities in (4) by $(v-u)^{+}$ and integrating over $\Omega$ we obtain

$$
\int_{\Omega}|\nabla v|^{p-2} \nabla v \cdot \nabla(v-u)^{+} d x+\int_{\Omega} g(x, v)(v-u)^{+} d x \leq 0
$$

and

Thus

$$
\int_{\Omega}|\nabla u|^{p-2} \nabla u \cdot \nabla(v-u)^{+} d x+\int_{\Omega} g(x, u)(v-u)^{+} d x \geq 0 .
$$

$$
\begin{gathered}
\int_{\Omega}\left(|\nabla v|^{p-2} \nabla v-|\nabla u|^{p-2} \nabla u\right) \cdot \nabla(v-u)^{+} d x \\
\leq \int_{\Omega}(g(x, u)-g(x, v))(v-u)^{+} d x \\
\quad=\int_{\{v>u\}}(g(x, u)-g(x, v))(v-u)^{+} d x .
\end{gathered}
$$

The last integral is non-positive since $g(x, \cdot)$ is non-decreasing. This in conjunction with

$$
\nabla(v-u)^{+}= \begin{cases}\nabla(v-u) & \text { if } v>u \\ 0 & \text { if } v \leq u\end{cases}
$$


yields

$$
\int_{\{v>u\}}\left(|\nabla v|^{p-2} \nabla v-|\nabla u|^{p-2} \nabla u\right) \cdot \nabla(v-u) d x \leq 0 .
$$

At this stage we recall the following result, whose proof can be found in [1, Lemma 2.1]: There exists $c>0$ such that for any $\eta, \eta^{\prime} \in \mathbb{R}^{N}$ with $|\eta|+\left|\eta^{\prime}\right|>0$, the following holds:

$$
\left(|\eta|^{p-2} \eta-\left|\eta^{\prime}\right|^{p-2} \eta^{\prime}\right) \cdot\left(\eta-\eta^{\prime}\right) \geq c\left(|\eta|+\left|\eta^{\prime}\right|\right)^{p-2}\left|\eta-\eta^{\prime}\right|^{2} .
$$

Applying (6) to (5) we find

$$
c \int_{\{v>u\}}(|\nabla u|+|\nabla v|)^{p-2}|\nabla u-\nabla v|^{2} d x \leq 0 .
$$

In order to derive a contradiction let us assume $\partial\{x \in \Omega: v(x)>u(x)\}$ intersects $\omega$, and consider $x_{0} \in \partial\{x \in \Omega: v(x)>u(x)\} \cap \omega$. From the hypotheses we infer $\frac{\partial u}{\partial \nu}\left(x_{0}\right)<0$. Since $u \in C^{1}(\bar{\Omega})$, it follows that there exists a positive constant $\gamma$ and an open set, relative to $\Omega$, denoted $V\left(x_{0}\right)$, containing $x_{0}$ such that $|\nabla u(x)| \geq \gamma$ for every $x \in V\left(x_{0}\right)$. Note that $x_{0} \in \partial W\left(x_{0}\right)$, where $W\left(x_{0}\right):=\{x \in \Omega: v(x)>$ $u(x)\} \cap V\left(x_{0}\right)$. From (7) we deduce that

$$
c \int_{W\left(x_{0}\right)}(|\nabla u|+|\nabla v|)^{p-2}|\nabla u-\nabla v|^{2} d x \leq 0 .
$$

Hence

$$
c \inf _{x \in W\left(x_{0}\right)}\{|\nabla u(x)|+|\nabla v(x)|\}^{p-2} \int_{W\left(x_{0}\right)}|\nabla(u-v)|^{2} d x \leq 0 .
$$

Since $|\nabla u|>\gamma$ on $W\left(x_{0}\right)$, it follows from the above inequality that

$$
c \gamma^{p-2} \int_{W\left(x_{0}\right)}|\nabla(u-v)|^{2} d x \leq 0
$$

so $\int_{W\left(x_{0}\right)}|\nabla(u-v)|^{2} d x=0$. This, in turn, implies that $u-v=$ const., in $W\left(x_{0}\right)$. Since $x_{0} \in \partial W\left(x_{0}\right)$, and $u\left(x_{0}\right)=v\left(x_{0}\right)$, we infer $u \equiv v$, in $W\left(x_{0}\right)$. However, this is not possible since $W\left(x_{0}\right) \subseteq\{x \in \Omega: v(x)>u(x)\}$. So, as desired, $\partial\{x \in \Omega: v(x)>u(x)\}$ does not intersect $\omega$.

Remark. It is worth noting that if $p \geq 2$, the condition $\frac{\partial u}{\partial \nu}<0$, on $\omega$, in Theorem 2 can be dropped. Indeed, in order to show that $\partial\{x \in \Omega: v(x)>u(x)\}$ does not intersect $\omega$, we proceed along the same lines as in the proof of the theorem until we reach (5). Then we use the following inequality whose proof like (6) can be found in [1, Lemma 2.1]: If $p \geq 2$, then there exists $\hat{c}>0$ such that for every $\eta, \eta^{\prime} \in \mathbb{R}$,

$$
\left(|\eta|^{p-2} \eta-\left|\eta^{\prime}\right|^{p-2} \eta^{\prime}\right) \cdot\left(\eta-\eta^{\prime}\right) \geq \hat{c}\left|\eta-\eta^{\prime}\right|^{p}
$$

Applying (8) to (5) yields

$$
\hat{c} \int_{\{v>u\}}|\nabla u-\nabla v|^{p} d x \leq 0,
$$

so $\int_{\{v>u\}}|\nabla u-\nabla v|^{p} d x=0$. Whence $u-v=$ const., on $\{x \in \Omega: v(x)>u(x)\}$. So if $x_{0} \in \partial\{x \in \Omega: v(x)>u(x)\} \cap \omega$, then $u \equiv v$ in $\{x \in \Omega: v(x)>u(x)\}$, which is false, and we have a contradiction.

We are now ready to prove Theorem 1 . As in the previous section we replace $u(h)$ by $u$. 
Proof of Theorem 2. We multiply the differential equation in (3) by $u$ and integrate the result over $D(h)$. Thus we obtain

(9)

$(p-1) \int_{D(h)}|\nabla u|^{p-2} \nabla u \cdot \nabla u^{\prime} d x=\lambda^{\prime}(h) \int_{D(h)} f u^{p} d x+(p-1) \lambda(h) \int_{D(h)} f u^{p-1} u^{\prime} d x$.

The right-hand side of (9) simplifies a great deal. Indeed from (1) we infer that the (domain) derivative of $\int_{D(h)} f u^{p} d x$ must be zero. Hence using [4, Theorem 3.3] we obtain

$$
\left(\int_{D(h)} f u^{p} d x\right)^{\prime}=p \int_{D(h)} f u^{p-1} u^{\prime} d x+\int_{\partial D(h)} f u^{p} V \cdot \nu d \sigma=0,
$$

where $d \sigma$ stands for the surface measure, and the "prime" denotes the domain derivative with respect to some vector field $V$. Since $u$ is zero on $\partial D(h)$ we deduce that $\int_{D(h)} f u^{p-1} u^{\prime} d x=0$, hence from (9) we find the following formula:

$$
(p-1)^{-1} \lambda^{\prime}(h)=\int_{D(h)}|\nabla u|^{p-2} \nabla u \cdot \nabla u^{\prime} d x,
$$

again thanks to (1). Let us recall the following characterization of the principal eigenvalue:

$$
\lambda(h)=\int_{D(h)}|\nabla u|^{p} d x .
$$

Hence, another application of [4, Theorem 3.3] yields

$$
\lambda^{\prime}(h)=p \int_{D(h)}|\nabla u|^{p-2} \nabla u \cdot \nabla u^{\prime} d x+\int_{\partial D(h)}|\nabla u|^{p} V \cdot \nu d \sigma .
$$

From (10) and (11) we find

$$
\lambda^{\prime}(h)=(1-p) \int_{\partial D(h)}|\nabla u|^{p} V \cdot \nu d \sigma .
$$

Let us choose a particular vector field as follows. Fix $\tilde{\phi} \in C_{0}^{\infty}(B)$ such that $0 \leq$ $\tilde{\phi} \leq 1$ and $\tilde{\phi} \equiv 1$ on a bounded neighborhood of $B_{h}$ inside $B$. The zero extension of $\tilde{\phi}$ to the whole of $\mathbb{R}^{N}$ is denoted by $\phi$. Finally we let $V:=\phi \vec{i}$, where $\vec{i}$ is the standard unit vector in $\mathbb{R}^{N}$. Obviously $V \in C^{2}\left(\mathbb{R}^{N} ; \mathbb{R}^{N}\right)$, so it can be inserted in (12). Thus we obtain

$$
\lambda^{\prime}(h)=(1-p) \int_{\partial B_{h}}|\nabla u|^{p} \nu_{1} d \sigma
$$

where $\nu_{1}$ is the first component of $\nu$. Note that since $p>1$ the assertion of the theorem follows once we establish that the integral in (13) is non-negative. To this end we shift the coordinate axes so that the hyperplane $l=\left\{x \in \mathbb{R}^{N}: x_{1}=0\right\}$ is positioned in such a way that it passes through the center of $B_{h}$, hence for $\left(x_{1}, y\right) \in B_{h}$ we have $\left(-x_{1}, y\right) \in B_{h}$, as well; that is, $B_{h}$ is symmetric with respect to $l$. Let us introduce some more notation. By $A_{l}$ we denote the part of $D(h)$ for which $x_{1} \geq 0$, and the image of $A_{l}$, with respect to $l$, is denoted $A_{l}^{*}$. Also $B_{h}^{+}$will stand for the part of $B_{h}$ for which $x_{1} \geq 0$, and $B_{h}^{-}$, the part of $B_{h}$ for which $x_{1} \leq 0$, in the new coordinate system. Next we introduce the function

$$
v(x)= \begin{cases}u(x) & \text { if } x \in A_{l}, \\ u\left(x_{l}\right) & \text { if } x \in A_{l}^{*},\end{cases}
$$


where $x_{l}$ stands for the reflection of $x$ about $l$. Observe that since $h \in\left[a_{0}, 1-a\right]$, $f(x)=-f^{-}(x)$, for $x \in A_{l}^{*}$. Therefore

$$
-\Delta_{p} u+\lambda f^{-}(x) u^{p-1}=0, \quad x \in A_{l}^{*} .
$$

On the other hand, using the definition of $v$, we have

$$
\begin{aligned}
-\Delta_{p} v(x)+\lambda f^{-}(x) v^{p-1}(x) & =-\Delta_{p} u\left(x_{l}\right)+\lambda f^{-}(x) u^{p-1}\left(x_{l}\right) \\
& \leq-\Delta_{p} u\left(x_{l}\right)+\lambda f^{-}\left(x_{l}\right) u^{p-1}\left(x_{l}\right) \\
& =-\Delta_{p} u\left(x_{l}\right)+\lambda f\left(x_{l}\right) u^{p-1}\left(x_{l}\right) \\
& =0,
\end{aligned}
$$

for $x \in A_{l}^{*}$. In the inequality above we used the fact that $f^{-}$is decreasing in the $x_{1}$-variable. So we have

$$
-\Delta_{p} v+\lambda f^{-}(x) v^{p-1} \leq 0, \quad x \in A_{l}^{*} .
$$

Thus we can apply Theorem 2 , with $g(x, z):=\lambda f^{-}(x) z^{p-1}, \omega:=\partial B_{h}^{-}$, to (14) and (15). Hence we infer existence of a neighborhood of $\partial B_{h}^{-}$, denoted $\mathcal{N}\left(\partial B_{h}^{-}\right)$, such that $u \geq v$ in $\mathcal{N}\left(\partial B_{h}^{-}\right)$. So, since $u$ and $v$ both vanish on $\partial B_{h}^{-}$, we obtain

$$
\frac{\partial}{\partial \nu}(u-v)(z)=\lim _{t \rightarrow 0^{-}} \frac{(u-v)(z+t \nu)-(u-v)(z)}{t} \leq 0, \quad z \in \partial B_{h}^{-},
$$

provided $t$ is small enough to ensure $z+t \nu \in \mathcal{N}\left(\partial B_{h}^{-}\right)$. From the above inequality we infer $\frac{\partial u}{\partial \nu}(z) \leq \frac{\partial v}{\partial \nu}(z)$, for $z \in \partial B_{h}^{-}$. However, since $\frac{\partial v}{\partial \nu}(z) \leq 0$, for $z \in \partial B_{h}^{-}$, it follows that $\left|\frac{\partial u}{\partial \nu}(z)\right| \geq\left|\frac{\partial v}{\partial \nu}(z)\right|$, for $z \in \partial B_{h}^{-}$. Note that for any $z \in \partial B_{h}^{-}$, we have $\nabla u(z)=-|\nabla u(z)| \nu(z)$. This, in turn, implies $\frac{\partial u}{\partial \nu}(z)=-|\nabla u(z)|$, so $\left|\frac{\partial u}{\partial \nu}(z)\right|=|\nabla u(z)|$, for $z \in \partial B_{h}^{-}$. Similarly one can show $\left|\frac{\partial v}{\partial \nu}(z)\right|=|\nabla v(z)|$, for $z \in \partial B_{h}^{-}$. Whence we derive $|\nabla u(z)| \geq|\nabla v(z)|$, for $z \in \partial B_{h}^{-}$. Thus

$$
\begin{aligned}
\int_{\partial B_{h}^{-}}|\nabla u(z)|^{p} \nu_{1}(z) d \sigma(z) & \geq \int_{\partial B_{h}^{-}}|\nabla v(z)|^{p} \nu_{1}(z) d \sigma(z) \\
& =\int_{\partial B_{h}^{-}}\left|\nabla u\left(z_{l}\right)\right|^{p} \nu_{1}(z) d \sigma(z) \\
& =\int_{\partial B_{h}^{+}}|\nabla u(z)|^{p} \nu_{1}\left(z_{l}\right) d \sigma(z) \\
& =-\int_{\partial B_{h}^{+}}|\nabla u(z)|^{p} \nu_{1}(z) d \sigma(z) .
\end{aligned}
$$

From this we get $\int_{\partial B_{h}^{-}}|\nabla u|^{p} \nu_{1} d \sigma \geq-\int_{\partial B_{h}^{+}}|\nabla u|^{p} \nu_{1} d \sigma$, hence $\int_{\partial B_{h}}|\nabla u|^{p} \nu_{1} d \sigma \geq$ 0 , as desired.

Remark. From Theorem 1, we infer that if the support of $f^{+}$is concentrated at $(-1,0) \in \mathbb{R} \times \mathbb{R}^{N-1}$, then $\lambda(h)$ is maximized when the two balls $B$ and $B_{h}$ are almost concentric.

\section{REFERENCES}

[1] L. Damascelli, Comparison theorems for some quasilinear degenerate elliptic operators and applications to symmetry and monotonicity results, Ann. Inst. Henri Poincaré, vol. 15, n. 4, 1998, p. 493-516. MR1632933 (99e:35081)

[2] M. Otani and T. Teshima, On the first eigenvalue of some quasilinear elliptic equations, Proc. Japan Acad. Ser. A Math. Sci., Vol. 64, no. 1, 1988, p. 8-10. MR953752 (89h:35257) 
[3] A.G. Ramm and P.N. Shivakumar, Inequalities for the minimal eigenvalue of the Laplacian in an annulus, Math. Inequal. Appl., Vol. 1, n. 4, 1998, p. 559-563. MR1646670 (99f:35149)

[4] J. Simon, Differentiation with respect to the domain in boundary value problems, Numer. Funct. Anal. and Optimiz., Vol. 2(7\&8), 1980, p. 649-687. MR619172 (83m:49032)

Department of Mathematics, The Petroleum Institute, P.O. Box 2533, Abu Dhabi, United Arab Emirates

E-mail address: bemamizadeh@pi.ac.ae

Department of Mathematics, Iran University of Science and Technology, Narmak, TEHRAN, IRAN

E-mail address: m_zivari@iust.ac.ir 